\title{
Development of Potent, Selective SRPK1 Inhibitors as Potential Topical Therapeutics for Neovascular Eye Disease
}

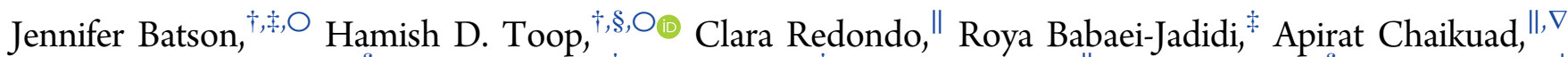
Stephen F. Wearmouth, ${ }^{\S}$ Brian Gibbons, ${ }^{\dagger}$ Claire Allen, ${ }^{\dagger}$ Cynthia Tallant, ${ }^{\|}$Jingxue Zhang, ${ }^{\S}$ Chunyun Du, ${ }^{\perp}$ Jules C. Hancox, ${ }^{\perp}$ Tom Hawtrey, ${ }^{\S}$ Joana Da Rocha, ${ }^{\S}$ Renate Griffith, ${ }^{\# \odot ~ S t e f a n ~ K n a p p, ~}{ }^{\circ, \nabla}$ David O. Bates, ${ }^{*},+\neq$ and Jonathan C. Morris*, ${ }^{*} \odot$

${ }^{\dagger}$ Exonate Ltd, Unit 23, Cambridge Science Park, Cambridge, United Kingdom

${ }^{\ddagger}$ Cancer Biology, Division of Cancer and Stem Cells, School of Medicine, University of Nottingham, Queen’s Medical Centre, Nottingham NG2 7UH, United Kingdom

${ }^{\S}$ School of Chemistry, UNSW Australia, Sydney, Australia

"Structural Genomic Consortium, University of Oxford, Old Road Campus, Oxford OX3 7DQ, United Kingdom

${ }^{\perp}$ School of Physiology and Pharmacology and Neuroscience, University of Bristol, BS8 1TD, Bristol, United Kingdom

${ }^{\#}$ School of Medical Sciences, UNSW Australia, Sydney, Australia

${ }^{\nabla}$ Institute for Pharmaceutical Chemistry and Buchmann Institute for Life Sciences, Johann Wolfgang Goethe-University, D-60438, Frankfurt am Main, Germany

\section{Supporting Information}

ABSTRACT: Serine/arginine-protein kinase 1 (SRPK1) regulates alternative splicing of VEGF-A to pro-angiogenic isoforms and SRPK1 inhibition can restore the balance of pro/ antiangiogenic isoforms to normal physiological levels. The lack of potency and selectivity of available compounds has limited development of SRPK1 inhibitors, with the control of alternative splicing by splicing factor-specific kinases yet to be translated. We present here compounds that occupy a binding pocket created by the unique helical insert of SRPK1, and trigger a backbone flip in the hinge region, that results in potent $(<10 \mathrm{nM})$ and selective inhibition of SRPK1 kinase activity. Treatment with these inhibitors inhibited SRPK1 activity and phosphorylation of serine/arginine splicing factor 1 (SRSF1), resulting in alternative splicing of VEGF-A from pro-angiogenic to antiangiogenic isoforms. This property resulted in potent inhibition of blood vessel growth in models of choroidal angiogenesis in vivo. This work identifies tool compounds for splice isoform selective targeting of pro-angiogenic VEGF, which may lead to new therapeutic strategies for a diversity of diseases where dysfunctional splicing drives disease development.
$\mathrm{V}$ ascular endothelial growth factor A (VEGF-A) is a key angiogenic factor in new blood vessel formation (angiogenesis $)^{1}$ and a critical driver of neovascular eye diseases ${ }^{2}$ including diabetic retinopathy, exudative (wet) age related macular degeneration (wAMD), and cancer progression. ${ }^{3}$ Many current clinical treatments, such as the monoclonal antibody Avastin (Bevacizumab) or antibody fragment Lucentis (ranibizumab), inhibit the action of VEGF-A in vivo. ${ }^{4}$ These broad anti-VEGF therapies are expensive, have the potential to generate significant cardiovascular and other off-target toxicities, and in eye disease must be administered by regular intraocular injection. There is, therefore, a need for a novel approach to VEGF-A inhibition that is cheaper, safer, and easier to administer.

VEGF-A actually exists as a number of isoforms, generated by alternative splicing of the eight-exon VEGFA gene, and these

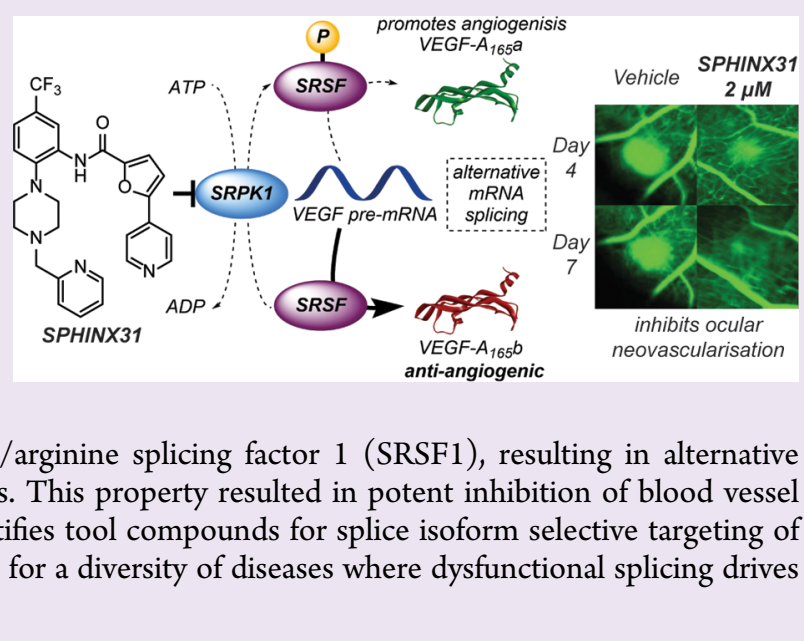

isoforms have different biological activities. ${ }^{5}$ VEGF-A premRNA can be alternatively spliced through a $3^{\prime}$ alternative splicing event in the terminal exon, generating isoforms with distinct and opposing functions. ${ }^{6}$ Proximal splice site (PSS) selection produces a pro-angiogenic isoform termed VEGF$\mathrm{A}_{165} \mathrm{a}$, whereas distal splice site (DSS) selection generates an antiangiogenic, but cytoprotective, isoform termed VEGF$\mathrm{A}_{165} \mathrm{~b}$. ${ }^{7}$ Angiogenesis occurs when the balance between pro- and antiangiogenic forms of VEGF-A is switched by differential splicing of the VEGF-A mRNA to the pro-angiogenic form. VEGF- $A_{165} b$ is endogenously expressed in normal tissues and downregulated in diabetic retinopathy and has been shown to

Received: November 27, 2016

Accepted: January 30, 2017

Published: January 30, 2017 
inhibit VEGF- $A_{165}$ a-mediated angiogenesis in physiological, pathological, and experimental settings. ${ }^{8}$

Serine/arginine (SR) proteins such as serine arginine-rich splicing factor 1 (SRSF1) regulate VEGF-A splicing by binding to the mRNA in a phosphorylation-dependent manner. ${ }^{9-11}$ Specific splice factor kinases are responsible for the phosphorylation of the SRSF proteins and can be used to modulate the splicing events ${ }^{10,11}$ For example, serine/arginineprotein kinase 1 (SRPK1) regulates splicing of pro-angiogenic VEGF-A $A_{165}$ a through phosphorylation of SRSF1, enabling SRSF1 nuclear translocation and binding to the proximal splice site in VEGF-A pre-mRNA. ${ }^{12}$

Modulation of this kinase can restore the balance of isoforms to normal, without harming cell function, ${ }^{10,11}$ particularly of the eye, ${ }^{13}$ as well as in cancer. ${ }^{14,15}$ Knockdown or inhibition of SRPK1 in models of neovascular eye disease, ${ }^{16}$ colorectal cancer, ${ }^{17}$ prostate cancer, ${ }^{18}$ or melanoma ${ }^{19}$ leads to distal splice site selection, generation of antiangiogenic VEGF- $\mathrm{A}_{165} \mathrm{~b}$, and reduced disease progression.

SRPK1 inhibitors have been proposed as potential therapeutics in angiogenic-related diseases, viral replication, and potential anticancer agents. However, existing classes of SRPK1 inhibitors, the (trifluoromethyl)phenylisonicotinamides, ${ }^{20}$ tricyclic quinoxaline derivatives, ${ }^{21}$ and 2-trifluoro-methyl-5,6-dihydro-7H-[1,3,4] thiadiazolo[3,2a]pyrimidin-7-ones, ${ }^{22}$ are neither specific nor potent. The discovery of quality leads with suitable pharmacological profiles for optimization and use as topical applications would be a significant advance.

Development of highly selective kinase inhibitors is challenging due to the high similarity of the ATP binding site that is usually targeted. ${ }^{23}$ Here, we describe experiments where we have taken advantage of combining a unique property of a specific insert close to the kinase hinge region in the SRPK family $^{24}$ with the presence of an unusual glycine residue that can allow a backbone flip, to rationally develop highly specific, very potent inhibitors of SRPK1 that are effective as eye drops in preventing neovascular eye disease in animal models.

\section{RESULTS AND DISCUSSION}

We recently identified a 3-(trifluoromethyl)anilide scaffold (SPHINX) with moderate potency but high selectivity for SRPK1 (Figure 1a,c). ${ }^{16}$ This inhibitor demonstrated that inhibition of SRPK allows for modulation of the VEGF-A splicing switch in vivo and in vitro. SPHINX was discovered through a screening campaign using differential scanning fluorimetry $(\mathrm{DSF})^{25}$ and in an in vitro kinase assay has an $\mathrm{IC}_{50}$ of $880 \mathrm{nM}$ for SRPK1. We investigated whether more potent molecules could be identified while maintaining target selectivity. Screening of a small series of related molecules identified compound $\mathbf{1}$, where the morpholine substituent was replaced with a piperazine moiety, which showed an $\mathrm{IC}_{50}$ of 31 $\mathrm{nM}$ (Figure 1a,c). A subsequent DSF screen against 55 kinases, which were selected based on cross reactivity of inhibitors targeting splicing kinases, showed that 1 interacted selectively with SRPK1 $\left(\Delta T_{\mathrm{m}} 8.54{ }^{\circ} \mathrm{C}\right)$ and SRPK3 $\left(\Delta T_{\mathrm{m}} 8.49{ }^{\circ} \mathrm{C}\right)$ over all of the other kinases tested, including SRPK2 $\left(\Delta T_{\mathrm{m}}<4{ }^{\circ} \mathrm{C}\right.$, Supporting Information Table 1).

To guide our inhibitor design, we solved a high resolution crystal structure of SRPK1 in complex with 1 (Figure 2). The structure of the complex provided a rationale for the selectivity of this inhibitor for the SRPK family. Structures of SRPKs revealed that a helix in the SRPK insertion domain packs
A<smiles>O=C(Nc1cc(C(F)(F)F)ccc1N1CCOCC1)c1ccc(I)o1</smiles>

SPHINX

B<smiles>[X]c1ccc(N2CC[Y]CC2)c(NC(=O)c2ccc([Z])o2)c1</smiles>

SPHINX scaffold<smiles>Cc1ccc(C(=O)Nc2cc(C(F)(F)F)ccc2N2CCN(C)CC2)o1</smiles><smiles>O=C(Nc1ccc(C(F)(F)F)cc1N1CCOCC1)c1ccc(-c2ccncc2)o1</smiles>

$2(\mathrm{Y}=\mathrm{NMe})$ $3\left(\mathrm{Y}=\mathrm{NCH}_{2} \mathrm{CH}_{2} \mathrm{NMe}_{2}\right)$ SPHINX31 $\left(Y=\mathrm{NCH}_{2}\right.$-2-pyridine)

\begin{tabular}{lccccc} 
C & \multicolumn{5}{c}{ Compound } \\
\cline { 2 - 6 } & SPHINX & $\mathbf{1}$ & $\mathbf{2}$ & $\mathbf{3}$ & SPHINX31 \\
\hline SRPK1 IC $50(\mathrm{nM})$ & 880 & 31 & 20 & 59 & 6 \\
\hline
\end{tabular}

D

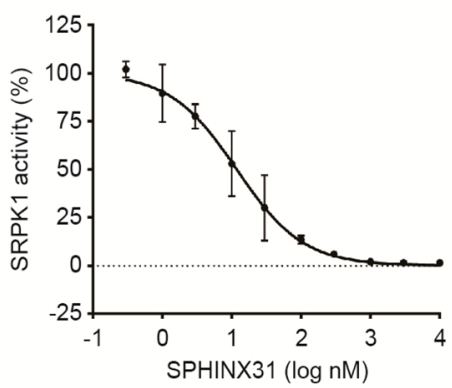

E

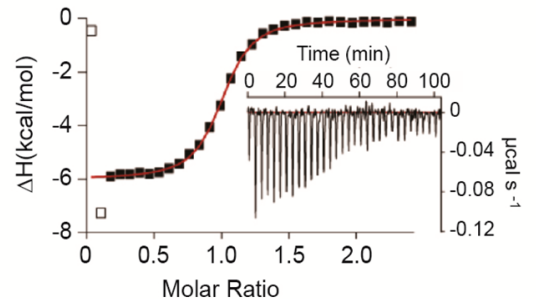

Figure 1. SPHINX compounds, inhibitors of SRPK1. (A) Structures of SPHINX and 1. (B) The SPHINX scaffold and structures of 2, 3, and SPHINX31. (C) IC 50 of compounds. (D) Radioactive SRPK1 kinase assay dose response with SPHINX31. (E) Isothermal calorimetry of SPHINX31 binding to SRPK1.

adjacent to the kinase hinge region, creating a unique hydrophobic pocket. The $\mathrm{CF}_{3}$ group of 1 was oriented toward this hydrophobic pocket, tightly interacting with this SRPK family specific binding cavity (Figure $2 c$ ).

The structure of SRPK1 in complex with 1 provided a model for a rational design of improved SRPK1 inhibitors. From examination of the binding site, we hypothesized that variation of $\mathrm{Y}$ and $\mathrm{Z}$ in the SPHINX scaffold (Figure 1b) with groups that could act as hydrogen-bond acceptors to interact with the catalytic lysine (K109) and/or the altered DFG (DLG) sequence in SRPK1 would improve potency, while maintaining interaction with the $\alpha$-helix of the SRPK insertion domain.

To this end, a small library of compounds was prepared from which we confirmed that replacement of the methyl substituent on the furan ring of $\mathbf{1}$ with a 4-pyridine group improved potency (i.e. compound 2, which is $20 \mathrm{nM}$ ), while further 


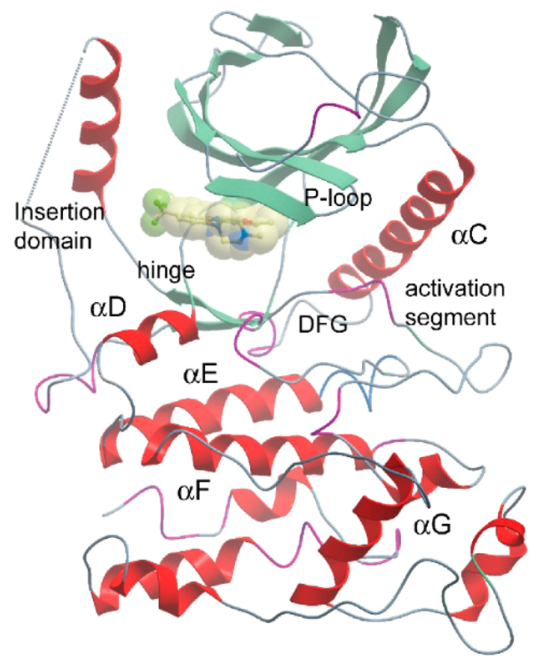

B

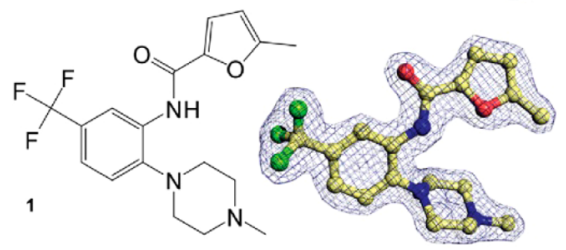

C

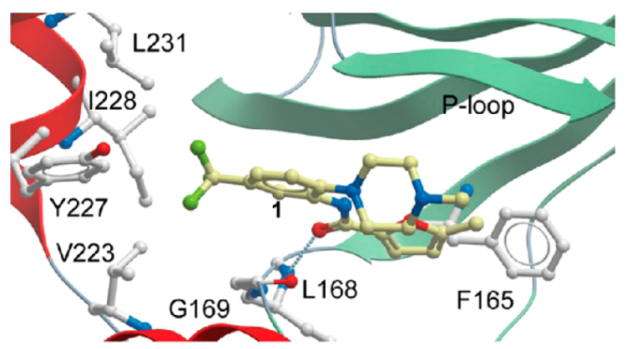

Figure 2. X-ray crystallography of $\mathbf{1}$ in SRPK1. (A) Co-crystallography of 1 in SRPK1 ( $\mathrm{pdb}$ id = 5MXX). (B) Structure of tool compound 1 (left panel) and $2 \mathrm{FoFc}$ electron density map around the ligand (right panel). (C) Ribbon structure showing $\mathrm{P}$ loop (green), 1, and highlighting the $\mathrm{CF}_{3}$ group interacting in the hydrophobic pocket located in the SRPK specific insert region.

improvement in potency was found by extending the substituent on the piperazine (Figure 1c). SPHINX31 emerged as a compound of excellent potency, with an $\mathrm{IC}_{50}$ of $5.9 \mathrm{nM}$ (Figure 1d), and we therefore chose this inhibitor as a chemical probe for further evaluation. Direct binding assays by isothermal titration calorimetry confirmed tight binding to SRPK1. The measured binding isotherm resulted in the determination of a dissociation constant of $72 \pm 5 \mathrm{nM}$ and a favorable binding enthalpy $(\Delta H:-5.98 \pm 0.2 \mathrm{kcal} / \mathrm{mol})$ and entropy (TSS: $3.9 \mathrm{kcal} / \mathrm{mol}$; Figure 1e).

The crystal structure of SPHINX31 with SRPK1 revealed structural features leading to the high potency and selectivity of this inhibitor (Figure 3). The binding mode of SPHINX31 was well-defined by electron density, and the structure was refined at $1.7 \AA$ resolution (Supporting Information Table 2). As observed in the structure of SRPK1 with 1 , the $\mathrm{CF}_{3}$ group interacted with the unique domain insert by binding to a deep hydrophobic cavity formed by the inset side chains of Y227, I228, and L231 and the hinge residue V167 (Figure 3b). The kinase catalytic domain assumed an active conformation as indicated by the presence of the canonical active site salt bridge (K109; E124) alignment of the hydrophobic spines and the "in" position of the altered DFG (DLG in SRPK1) motif. The 4-

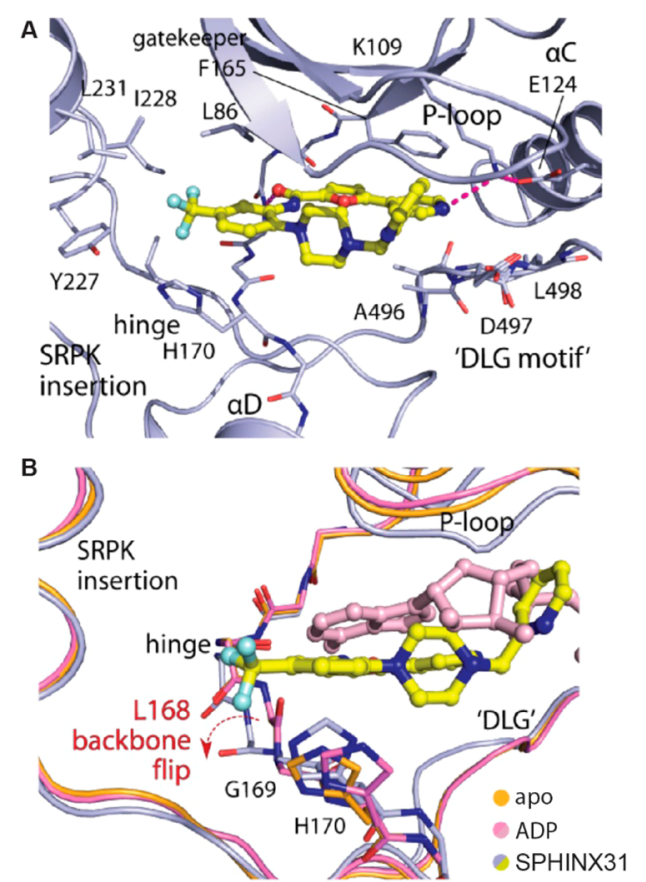

Figure 3. X-ray crystallography of SPHINX31 in SRPK1. (A) Cocrystal structure of SPHINX31 bound to SRPK1 (pdb id = 5MY8). (B) Overlay of hinge region of unbound (orange) and SPHINX31bound (gray) SRPK1. It can be seen that the trifluoromethyl group (cyan) interacts with the glycine to flip the hinge outward (arrow). The ATP binding pocket of SPHINX31 unbound (orange) and SPHINX31-bound (gray) SRPK1 shows that the phosphorylation (P)loop is substantially pushed upward by SPHINX31.

pyridine group attached to the furan projects into the binding site, interacting with E124. In addition, the 4-pyridine moiety stacks against the aromatic sides chain of the gatekeeper (F165). The pyridine attached to the piperazine ring orients toward the P-loop, accommodating a hydrophobic pocket formed by V94, S92, and P-loop main chain atoms. The improved contacts, in particular the aromatic stacking interactions, provide a rationale for the significantly improved inhibitor potency of SPHINX31 when compared with $\mathbf{1}$.

When compared with the SRPK1 apo-structure, the SPHINX31 complex revealed several inhibitor-induced changes. Most significantly, the backbone carbonyl of the hinge residue L168 flipped to make room for the trifluoromethyl group (Figure $3 \mathrm{~b}$ ). The phosphate binding loop (Ploop) also significantly changed conformation when compared with the structure of the apo-kinase domain. This loop region across kinases is usually very dynamic, but in SRPK1 it is unusually rich in aromatic residues, which normally results in decreased movement. However, here, the P-loop is distorted to allow tighter contact of side chain residues with the inhibitor.

In the kinase hinge region, structural change induced by flipping of the backbone carbonyl of the hinge residue is facilitated by the presence of the glycine residue (G169) that introduces flexibility in this region. Glycine residues are rare in hinge regions, but similar backbone flips have been evoked as the structural mechanism explaining the excellent selectivity of the p38 inhibitor skepionone $\mathrm{L}^{26} \mathrm{~A}$ similar backbone flip has also been observed in the recent report ${ }^{27}$ of SRPIN340, supporting our hypothesis that this structural change offers an interesting opportunity for the design of inhibitor selectivity. 

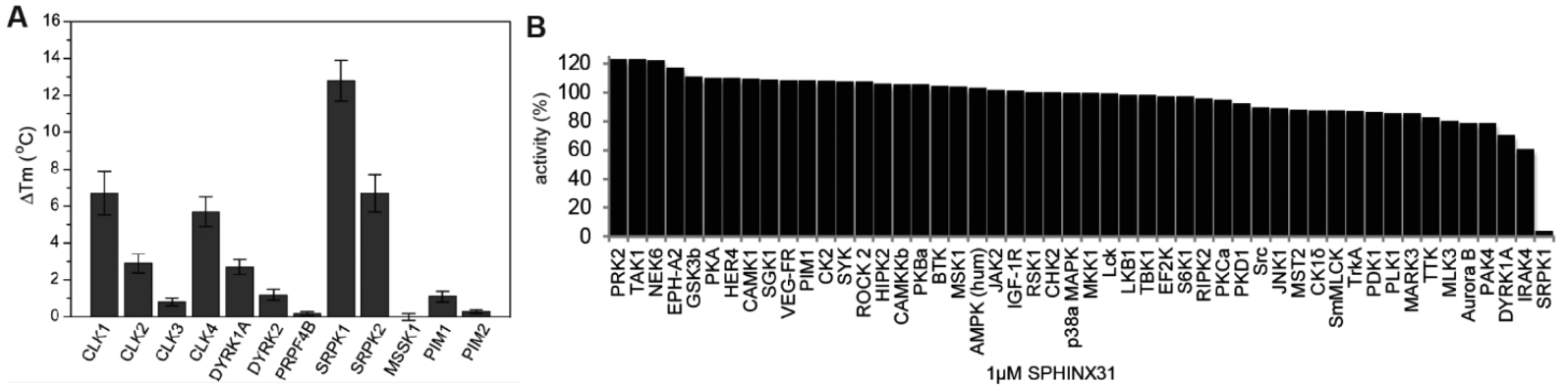

Figure 4. SPHINX31, highly selective for SRPK1. (A) Differential scanning fluorimetry temperature shift of SPHINX31 against SRPK1 and closely related kinases. (B) International Centre for Kinase Profiling screen of SPHINX31 against a series of key 50 enzymes that provide representative sampling of the human kinome.

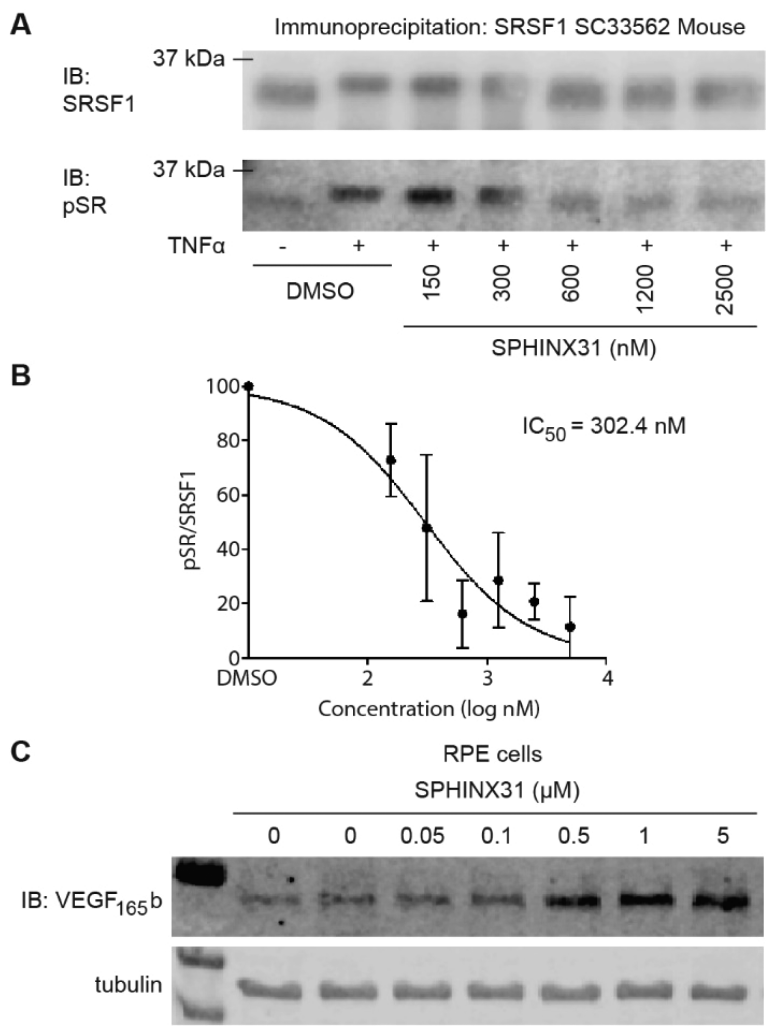

Figure 5. Inhibition of cellular SRPK1 activity. (A) Dose response of SPHINX31 inhibits TNF $\alpha$-mediated SRSF1 phosphorylation. Immunoprecipitation with SRSF1 antibody and immunoblotting with phosphoSR in PC-3 cells to detect phosphorylated SRSF1 $(n=3$ independent experiments). (B) Quantitation of the westen blot bands shown in panel A. The ratio between phosphor and unmodified SRSF1 is shown. Quantification of $\mathrm{pSR} / \mathrm{SRSF} 1$ ratio indicates an $\mathrm{IC}_{50}$ of 367.3 $\mathrm{nM}$ for SPHINX31. (C) Dose response with SPHINX31 and immunoblotting for VEGF165b in RPE cells. Tubulin is used to control for equal loading. $n=3$ dose response inhibitor treatments.

The SPHINX scaffold has high selectivity ${ }^{19}$ for SRPK1 over SRPK2, and this selectivity can be rationalized by the differences in the helical insert region, with the key residues in this region for SRPK1 being Y227, I228, and L231, while in SRPK2, I228 is replaced by a valine (V240) and L231 is replaced by a methionine (M243). We were able to solve a crystal structure of SRPK2 in complex with 1 , and as can be seen in the overlay of the two crystal structures SPHINX31.SRPK1 and 1.SRPK2 (Supporting Information Figure 1), the methionine residue is significantly larger than
A

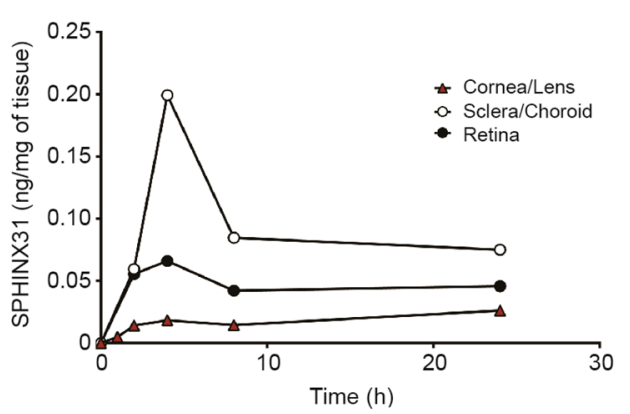

B

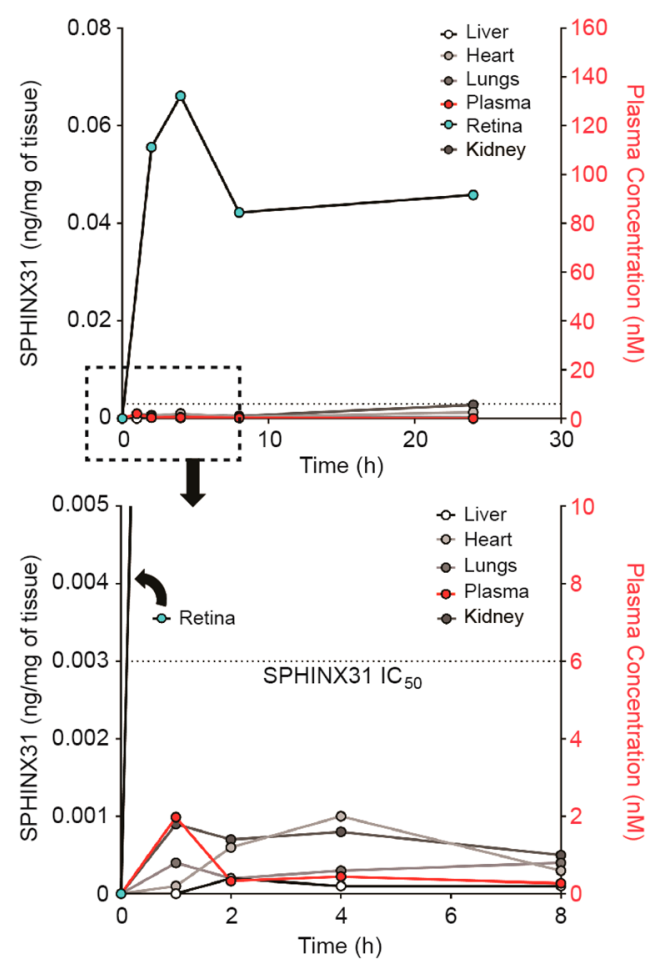

Figure 6. Ocular penetration of SPHINX31. (A) Amount (ng per mg of tissue) of SPHINX31 following single $0.05 \mu \mathrm{g} / 10 \mu \mathrm{L}$ eye drop administration in the indicated eye tissue and (B) in plasma and systemic organs. Dashed line is $\mathrm{IC}_{50}$ on plasma axis. The inset shows a blowup of the bottom left-hand corner of the graph showing detectable SPHINX31 in plasma at low concentration.

the leucine residue in SRPK1, which makes the pocket in SRPK2 smaller and results in a slight reorientation of the inhibitor and the $\mathrm{CF}_{3}$ group. As a consequence, 1 cannot bind as tightly to SRPK2 as indicated by the lengthening of the $\mathrm{H}$ bond between V167 and the amido group (1.82 A versus 1.72 
A

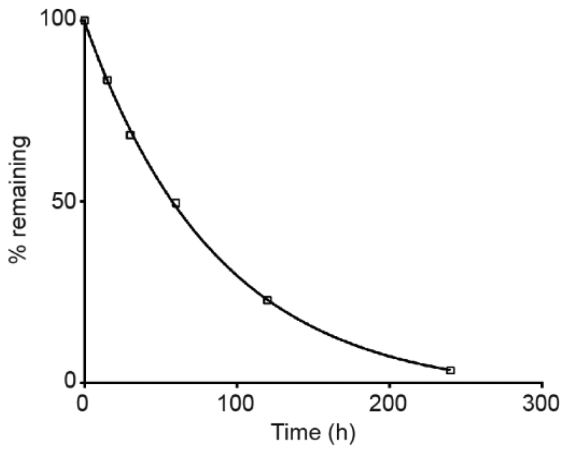

B

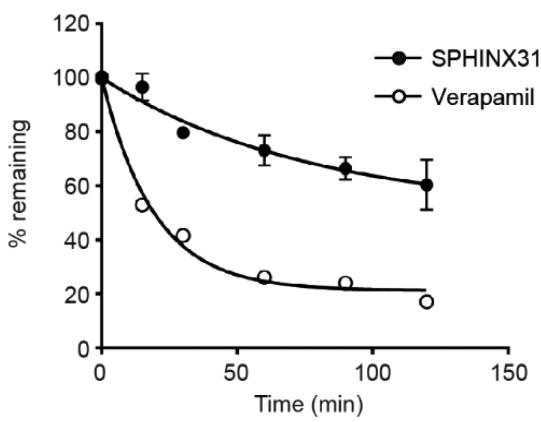

Figure 7. Metabolic stability of SPHINX31. (A) \% SPHINX31 remaining at the indicated time points in mouse plasma after incubation at $37{ }^{\circ} \mathrm{C}$. (B) \% SPHINX31 remaining at the indicated time points after incubation with mouse liver microsomes.

$\AA$ ) and the distance between $\mathrm{Y} 227$ and the $\mathrm{CF}_{3}$ group ( $3.7 \AA$ in SRPK2; 3.3 A in SRPK1).

Due to their role in splicing, we were particularly interested in the activity of the developed inhibitors on all of the splicing modulating kinases. We therefore used differential scanning fluorimetry to screen SPHINX31 against this subgroup of kinases; CLK family (CLK1, -2, -3, -4), DYRK1A and DYRK2 as well as PRBF4B (DYRK5), the SRPK family (SRPK1, -2 , and -3 ), and the PIM1 and PIM2 kinases, which we have found often interact with splicing kinase inhibitors. As expected, the $\Delta T_{\mathrm{m}}$ data showed strong interaction for SRPK1 $\left(\Delta T_{\mathrm{m}}\right.$ of 12.8 $\left.{ }^{\circ} \mathrm{C}\right)$; weak binding to SRPK2, CLK1 $\left(\Delta T_{\mathrm{m}}\right.$ of $\left.6.7^{\circ} \mathrm{C}\right)$, and CLK4 $\left(\Delta T_{\mathrm{m}}\right.$ of $\left.5.7^{\circ} \mathrm{C}\right)$; and no significant $\Delta T_{\mathrm{m}}$ shifts for other splicing kinases (Figure 4a). This degree of difference in temperature shift $\left(6.1^{\circ} \mathrm{C}\right.$ for SRPK2 and CLK1 compared with SRPK1, and $7.1{ }^{\circ} \mathrm{C}$ for CLK4) equates to a difference in binding affinity of 50-fold for SRPK2 and CLK1 and 100-fold for CLK $4,{ }^{25}$ confirming the high selectivity of SPHINX31 for SRPK1.

Kinase assays showed that SPHINX31 was a type 1 kinase inhibitor (ATP competitive, Supporting Information Figure 2). A radiolabeled ATP competition assay was carried out against 50 kinases, which have been selected by the International Centre for Kinase Profiling as being representative of the human kinome, including SRPK1. This demonstrated 96\% inhibition of SRPK1 activity at $1 \mu \mathrm{M}$ by SPHINX31, but no other kinase was significantly inhibited in that panel (Figure $4 \mathrm{~b})$.

To determine whether SPHINX31 inhibits SRPK1 activity in cells, PC3 prostate cancer cells (previously been shown to have high SRPK1 mediated SRSF1 phosphorylation ${ }^{18}$ ) were treated with SPHINX31. This resulted in inhibition of SRSF1 phosphorylation at an inhibitor concentration of $300 \mathrm{nM}$ (Figure 5a,b). Quantitative analysis of the Western blot bands

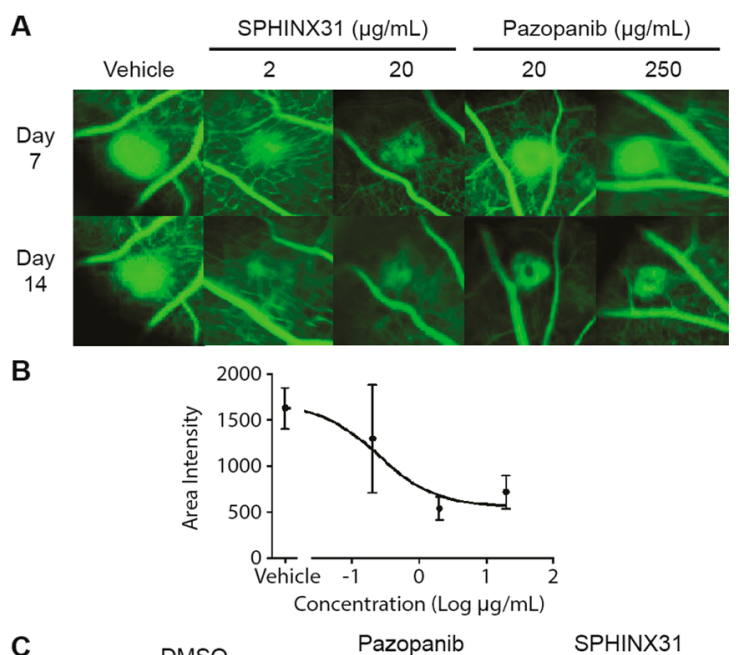

C

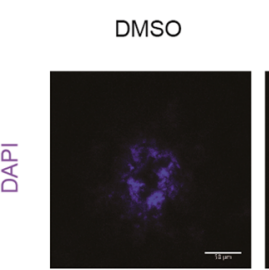

$20 \mu \mathrm{g} / \mathrm{mL}$ $2 \mu \mathrm{g} / \mathrm{mL}$
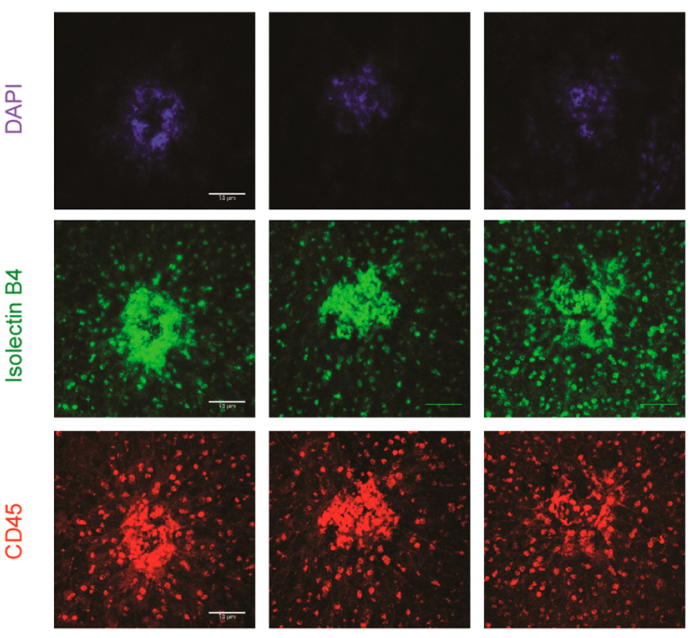

D

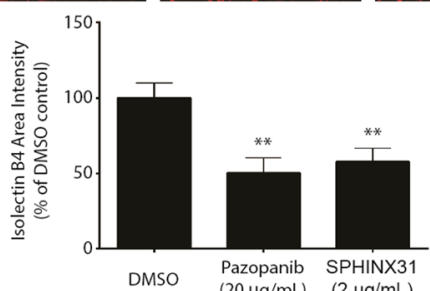

E

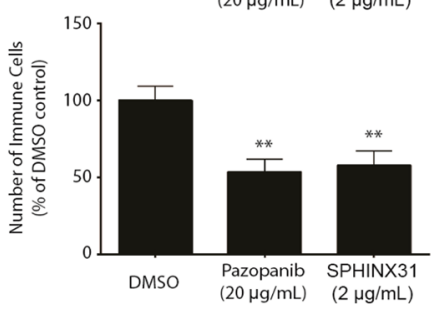

Figure 8. Antiangiogenic efficacy of SRPK1 inhibitor eye drops. (A) Fluorescein angiography representative images at day 7 and day 14 after laser CNV induction in C57/B16 mice with vehicle, SPHINX31 $(2 \mu \mathrm{g} / \mathrm{mL}$ or $20 \mu \mathrm{g} / \mathrm{mL})$ and pazopanib $(20 \mu \mathrm{g} / \mathrm{mL}$ and $250 \mu \mathrm{g} / \mathrm{mL})$. (B) Quantification of area $\times$ intensity for SPHINX31. $N$ (lesion) $=$ 152 (DMSO), 36 (SPHINX31, $0.2 \mu \mathrm{g} / \mathrm{mL}$ ), 36 (SPHINX31, $2 \mu \mathrm{g} /$ $\mathrm{mL}$ ), 46 (SPHINX31, $20 \mu \mathrm{g} / \mathrm{mL}$ ). (C) RPE/choroid flatmount Imaris representative images for DAPI, isolectin $\mathrm{B} 4$, and CD45 immunofluorescence after 14 days treatment with DMSO, pazopanib $(20 \mu \mathrm{g} /$ $\mathrm{mL})$, or SPHINX31 $(2 \mu \mathrm{g} / \mathrm{mL})$. (D) Quantification of isolectin B4 area $X$ intensity (\% DMSO controls). (E) Number of immune cells (\% DMSO controls). $* * p<0.01$, Kruskall-Wallis ANOVA $(N$ (lesion $)=$ 30 (DMSO), 12 (SPHINX31, $2 \mu \mathrm{g} / \mathrm{mL}$ ), 16 (Pazopanib, $20 \mu \mathrm{g} / \mathrm{mL}$ )). 
revealed an EC50 of about $360 \mathrm{nM}$ (Figure 5b). The effect on downstream splicing activity was also investigated in retinal pigmented epithelials (RPE, a major source of VEGF in the retina). The compounds dose-dependently switched splicing from VEGF-A $A_{165}$ a to VEGF-A $A_{165} b$ in RPE cells (Figure $5 c$ ).

The VEGF- $\mathrm{A}_{165} \mathrm{~b}$ antibody detects the $\mathrm{C}$-terminal sequence of VEGF encoded by exon $8 \mathrm{~b}$. A recent report ${ }^{28}$ showed that it is possible to generate a form of VEGF by post-translation readthrough (PTR), where the stop codon in exon8a is repressed, resulting in an extended VEGF (VEGF-Ax), which is antiangiogenic and can be detected by the VEGF- $\mathrm{A}_{165} \mathrm{~b}$ antibodies. To determine whether SPHINX31 was causing splicing to VEGF- $\mathrm{A}_{165} \mathrm{~b}$ and not production of VEGF-Ax by PTR, an immunoblot of RPE cells was undertaken with a VEGFAx specific antibody. VEGF-Ax was not found in untreated RPE cells (Supporting Information Figure 3).

We had previously shown that SRPK1 inhibition by SRPIN340 $\left(\mathrm{IC}_{50} 0.96 \mu \mathrm{M}\right)$ or SPHINX $\left(\mathrm{IC}_{50} 0.88 \mu \mathrm{M}\right)$ was antiangiogenic in mouse models of choroidal neovascularisation, as eye drops with a maximum effect at $10 \mu \mathrm{g} / \mathrm{mL}(28.5$ $\mu \mathrm{M}) .{ }^{16}$ To determine whether more potent molecules retain ocular penetration, we measured the amount of SPHINX31 retained in ocular compartments after application to the surface of the eye in eye drops. A total of $0.05 \mu \mathrm{g}$ of SPHINX31 was applied to the left eyes of 12 mice; then the animals were sacrificed and samples collected at $1,4,8$, and $24 \mathrm{~h}(N=1$ per time point). Retinae were dissected from the scleral/choroidal complex and SPHINX31 measured. Figure 6A shows that SPHINX31 was found in retinae and sclera/choroid at similar levels, equivalent to approximately $0.24 \%$ (retina) and $0.43 \%$ (sclera/choroid) of the starting dose in these tissues at $4 \mathrm{~h}$ after application of the eye drop. Figure 6B shows that there was an initial low spike in SPHINX31 in plasma at $1 \mathrm{~h}(2 \mathrm{nM})$, which was lost by $2 \mathrm{~h}$ and detectable at only very low levels at all other time points. In the kidney and heart, trace amounts $(<0.01 \mathrm{ng} /$ mg tissue) of SPHINX31 could be detected only after $24 \mathrm{~h}$.

To determine stability, we measured levels of SPHINX31 in mouse plasma after incubation at $37^{\circ} \mathrm{C}$. Figure $7 \mathrm{~A}$ shows that SPHINX31 is rapidly broken down. Metabolic stability in mouse liver microsomes showed that SPHINX31 had medium clearance with a $T_{1 / 2}$ of $95.79 \mathrm{~min}$ compared to the positive control Verapamil $T_{1 / 2}$ of $11.15 \mathrm{~min}$ (Figure $7 \mathrm{~B}$ ). Rapid clearance can improve the therapeutic window through reduced risk of off-target effects, and this is an important consideration in ongoing research for both eye drop administration and development of systemic therapeutics.

Cell-based assays of cytotoxicity (Supporting Information Figure 4) were carried out for the compounds that were potent inhibitors. Compound 3 had an $\mathrm{EC}_{50}$ of $10-50 \mu \mathrm{M}$, whereas the $\mathrm{EC}_{50}$ for 1 and SPHINX31 was larger than $100 \mu \mathrm{M}$, and 1 and SPHINX31 showed $<5 \%$ toxicity at $100 \mu \mathrm{M}$. Ames tests indicated that neither SPHINX31 nor its breakdown product showed any genotoxicity. Addition of SPHINX31 as an eye drop to mice demonstrated no alteration in electro-retinogram signals (Supporting Information Figure 5). We also tested whether SPHINX31 could inhibit the human ether a-go-go related gene (hERG) potassium channel using patch clamp electrophysiology. While SPHINX did not inhibit hERG, as we had previously described, ${ }^{16}$ we found that compound 3 did, with an $\mathrm{IC}_{50}$ of $36 \mathrm{nM}$. In contrast, SPHINX31 inhibited hERG with an $\mathrm{IC}_{50}$ of $300 \mathrm{nM}$ (Supporting Information Figure 6). This is 50-fold higher than its IC $_{50}$ value against SRPK1 (5.9 $\mathrm{nM}$ ), so, combined with relatively rapid clearance, it is believed that hERG inhibition is not a significant issue for this compound.

Having established that SPHINX31 could penetrate into the eye and that it was not toxic, we therefore tested the effect of SPHINX31 as an eyedrop in a mouse model of choroidal neovascularization, which models wet age-related macular degeneration (Figure 8A). 22,23 SPHINX31 exerted a dose dependent inhibition of choroidal neovascularisation (Figure $8 \mathrm{~B})$, with similar efficacy at $2 \mu \mathrm{g} / \mathrm{mL}(3.8 \mu \mathrm{M})$ to that achieved by SPHINX or SRPIN340 at $10 \mu \mathrm{g} / \mathrm{mL}(28.5 \mu \mathrm{M})$, and greater effect at $2 \mu \mathrm{g} / \mathrm{mL}$ than pazopanib at $250 \mu \mathrm{g} / \mathrm{mL}(572 \mu \mathrm{M})$. Staining of choroid for endothelial cells (isolectin-B4) and infiltrating macrophages (CD45) demonstrated that both pazopanib and SPHINX31 inhibited blood vessel growth and macrophage infiltration (Figure 8C,D). The level of inhibition of lesion size $(\sim 50 \%)$ is similar to that seen with inhibitors of VEGF (e.g., VEGF-TRAP, $30 \%){ }^{28}$ recombinant VEGF-A ${ }_{165}$ b protein $(43 \%),{ }^{29}$ or intraocular injection of SRPK1 inhibitors ${ }^{6,19,21}$ Extraction of protein from the retinae of the animals demonstrated VEGF- $\mathrm{A}_{165} \mathrm{~b}$ expression in the retinae, which was greater in treated than in nontreated eyes measured both by Western blot (Supporting Information Figures 7A,B) and by isoform specific ELISA, where a switch in splicing was seen toward antiangiogenic VEGF (Supporting Information Figure $7 \mathrm{C}-\mathrm{E}$ ). Coupled with our earlier observation of the penetration of SPHINX31 into the retina, we believe that these data show that SPHINX31 represents a good lead for the development of antiangiogenic therapeutics in the eye.

In summary, we have shown here the development of highly potent, selective, and cell active SRPK1 inhibitors. Importantly, these compounds also have antiangiogenic properties in vivo. These compounds occupy a binding pocket created by the unique helical insert of SRPK1 and trigger a backbone flip in the hinge region that results in potent $(<10 \mathrm{nM})$ and selective inhibition of SRPK1 kinase activity. SPHINX31 emerged as the most optimal inhibitor of a series of inhibitors that were synthesized. SPHINX31 showed good cellular activity, promising pharmacokinetic properties and potency in in vivo models of angiogenesis. SPHINX31 will facilitate the development of topical therapeutics for neovascular eye disease and provide validation of SRPK1 as a target for drug development in multiple diseases.

\section{METHODS}

All of the methods and procedures are presented in the Supporting Information.

\section{ASSOCIATED CONTENT}

\section{S Supporting Information}

The Supporting Information is available free of charge on the ACS Publications website at DOI: 10.1021/acschembio.6b01048.

Methods, tables, and figures (PDF)

\section{AUTHOR INFORMATION}

\section{Corresponding Authors}

*E-mail: David.Bates@nottingham.ac.uk.

*E-mail: jonathan.morris@unsw.edu.au.

ORCID

Hamish D. Toop: 0000-0003-4637-4764

Renate Griffith: 0000-0001-7739-5686

Jonathan C. Morris: 0000-0002-5109-9069 


\section{Author Contributions}

OAuthors contributed equally. J.C.M., D.O.B., and S.K. conceived the project, designed experiments, analyzed data, and wrote the manuscript with input from all coauthors. J.C.M. conceived and directed the chemistry effort. H.D.T., S.F.W., J.Z., T.H., J.D.R., and R.G. performed modeling, chemical synthesis, and characterization. D.O.B. conceived and directed the biological experimental research. J.B., R.B.-J., B.G., C.A., C.D., and J.H. carried out biological experimental research analysis. C.R., A.C., and C.T. performed X-ray crystallography research and analysis, DSF screening, and isothermal calorimetry.

\section{Notes}

The authors declare the following competing financial interest(s): D.O.B is founder and shareholder of Exonate Ltd. J.C.M. is a consultant to Exonate Ltd. J.C.M. and S.K are members of the Scientific Advisory Board for Exonate Ltd.

\section{ACKNOWLEDGMENTS}

This work was funded by the MRC (MR/K020366/1, DOB), the University of Nottingham HERMES fellowship (J.B.), UNSW Australia (Goldstar Scheme), and Exonate Ltd, UK. A.C. is grateful for support by the EU network grant PRIMES. C.D. and J.H. funded by Heart Research UK HRUK RG2594/ $11 / 13$. S.K. is grateful for support by the SGC, a registered charity (number 1097737) that receives funds from AbbVie, Bayer Pharma AG, Boehringer Ingelheim, Canada Foundation for Innovation, Eshelman Institute for Innovation, Genome Canada through Ontario Genomics Institute, Janssen, Merck \& Co., Novartis Pharma AG, Ontario Ministry of Economic Development and Innovation, Pfizer, São Paulo Research Foundation-FAPESP, Takeda, and the Wellcome Trust.

\section{REFERENCES}

(1) Hoeben, A., Landuyt, B., Highley, M. S., Wildiers, H., Van Oosterom, A. T., and De Bruijn, E. A. (2004) Vascular endothelial growth factor and angiogenesis. Pharmacological Rev. 56, 549-580.

(2) Penn, J. S., Madan, A., Caldwell, R. B., Bartoli, M., Caldwell, R. W., and Hartnett, M. E. (2008) Vascular endothelial growth factor in eye disease. Prog. Retinal Eye Res. 27, 331-371.

(3) Folkman, J., Merler, E., Abernathy, C., and Williams, G. (1971) Isolation of a tumor factor responsible for angiogenesis. J. Exp. Med. $133,275-288$.

(4) Niu, G., and Chen, X. (2010) Vascular endothelial growth factor as an anti-angiogenic target for cancer therapy. Curr. Drug Targets 11, $1000-1017$.

(5) Houck, K. A., Leung, D. W., Rowland, A. M., Winer, J., and Ferrara, N. (1992) Dual regulation of vascular endothelial growth factor bioavailability by genetic and proteolytic mechanisms. J. Biol. Chem. 267, 26031-26037.

(6) Amin, E. M., Oltean, S., Hua, J., Gammons, M. V. R., HamdollahZadeh, M., Welsh, G. I., Cheung, M.-L., Ni, L., Kase, S., Rennel, E. S., Symonds, K. E., Nowak, D. G., Royer-Pokora, B., Saleem, M. A., Hagiwara, M., Schumacher, V. A., Harper, S. J., Hinton, D. R., Bates, D. O., and Ladomery, M. R. (2011) WT1 mutants reveal SRPK1 to be a downstream angiogenesis target by altering VEGF splicing. Cancer Cell 20, 768-780.

(7) Harper, S. J., and Bates, D. O. (2008) VEGF-A splicing: the key to anti-angiogenic therapeutics? Nat. Rev. Cancer 8, 1250-1257.

(8) Artac, R. A., McFee, R. M., Longfellow Smith, R. A., BaltesBreitwisch, M. M., Clopton, D. T., and Cupp, A. S. (2009) Neutralization of vascular endothelial growth factor antiangiogenic isoforms is more effective than treatment with proangiogenic isoforms in stimulating vascular development and follicle progression in the perinatal rat ovary. Biol. Reprod. 81, 978-988.
(9) (a) Sanford, J. R., Ellis, J. D., Cazalla, D., and Caceres, J. F. (2005) Reversible phosphorylation differentially affects nuclear and cytoplasmic functions of splicing factor 2/alternative splicing factor. Proc. Natl. Acad. Sci. U. S. A. 102, 15042-15047. (b) Karni, R., de Stanchina, E., Lowe, S. W., Sinha, R., Mu, D., and Krainer, A. R. (2007) The gene encoding the splicing factor SF2/ASF is a proto-oncogene. Nat. Struct. Mol. Biol. 14, 185-193.

(10) Nowak, D. G., Amin, E. M., Rennel, E. S., Hoareau-Aveilla, C., Gammons, M., Damodoran, G., Hagiwara, M., Harper, S. J., Woolard, J., Ladomery, M., and Bates, D. O. (2010) Regulation of vascular endothelial growth factor (VEGF) splicing from pro-angiogenic to anti-angiogenic isoforms: a novel therapeutic strategy for angiogenesis. J. Biol. Chem. 285, 5532-5540.

(11) Nowak, D. G., Woolard, J., Amin, E. M., Konopatskaya, O., Saleem, M. A., Churchill, A. J., Ladomery, M. R., Harper, S. J., and Bates, D. O. (2008) Expression of pro- and anti-angiogenic isoforms of VEGF is differentially regulated by splicing and growth factors. J. Cell Sci. 121, 3487-3495.

(12) Oltean, S., Gammons, M., Hulse, R., Hamdollah-Zadeh, M., Mavrou, A., Donaldson, L., Salmon, A. H., Harper, S. J., Ladomery, M. R, and Bates, D. O. (2012) SRPK1 inhibition in vivo: modulation of VEGF splicing and potential treatment for multiple diseases. Biochem. Soc. Trans. 40, 831-835.

(13) Ergorul, C., Ray, A., Huang, W., Darland, D., Luo, Z. K., and Grosskreutz, C. L. (2008) Levels of vascular endothelial growth factorA165b (VEGF-A165b) are elevated in experimental glaucoma. Mol. Vis. 14, 1517-1524.

(14) Merdzhanova, G., Gout, G. S., Keramidas, M., Edmond, V., Coll, J. L., Brambilla, C., Brambilla, E., Gazzeri, S., and Eymin, B. (2010) The transcription factor E2F1 and the SR protein SC35 control the ratio of pro-angiogenic versus antiangiogenic isoforms of vascular endothelial growth factor-A to inhibit neovascularization in vivo. Oncogene 29, 5392-5403.

(15) Li, Z. Y., Zhu, F., Hu, J. L., Peng, G., Chen, J., Zhang, S., Chen, X., Zhang, R. G., Chen, L. I., Liu, P., Luo, M., Sun, Z. H., Ren, J. H., Huang, L. L., and Wu, G. S. (2011) Sp1 inhibition-mediated upregulation of VEGF165b induced by rh-endostatin enhances antiangiogenic and anticancer effect of rh-endostatin in A549. Tumor Biol. 32, 677-687.

(16) Gammons, M. V., Fedorov, O., Ivison, D., Du, C., Clark, T., Hopkins, C., Hagiwara, M., Dick, A. D., Cox, R., Harper, S. J., Hancox, J. C., Knapp, S., and Bates, D. O. (2013) Topical Antiangiogenic SRPK1 Inhibitors Reduce Choroidal Neovascularization in Rodent Models of Exudative AMD. Invest. Ophthalmol. Visual Sci. 54, 60526062.

(17) Hayes, G. M., Carrigan, P. E., and Miller, L. J. (2007) Serinearginine protein kinase 1 overexpression is associated with tumorigenic imbalance in mitogen-activated protein kinase pathways in breast, colonic, and pancreatic carcinomas. Cancer Res. 67, 2072-2080.

(18) Mavrou, A., Brakspear, K., Hamdollah-Zadeh, M., Damodaran, G., Babaei-Jadidi, R., Oxley, J., Gillatt, D. A., Ladomery, M. R., Harper, S. J., Bates, D. O., and Oltean, S. (2015) Serine-arginine protein kinase 1 (SRPK1) inhibition as a potential novel targeted therapeutic strategy in prostate cancer. Oncogene 34, 4311-4319.

(19) Gammons, M. V., Lucas, R., Dean, R., Coupland, S. E., Oltean, S., and Bates, D. O. (2014) Targeting SRPK1 to control VEGFmediated tumour angiogenesis in metastatic melanoma. Br. J. Cancer $111,477-485$.

(20) Fukuhara, T., Hosoya, T., Shimizu, S., Sumi, K., Oshiro, T., Yoshinaka, Y., Suzuki, M., Yamamoto, N., Herzenberg, L. A., Herzenberg, L. A., and Hagiwara, M. (2006) Utilization of host SR protein kinases and RNA-splicing machinery during viral replication. Proc. Natl. Acad. Sci. U. S. A. 103, 11329-11333.

(21) Székelyhidi, Z., Pató, J., Wáczek, F., Bánhegyi, P., HegymegiBarakonyi, B., Erôs, D., Mészáros, G., Hollósy, F., Hafenbradl, D., Obert, S., Klebl, B., Kéri, G., and Őrfi, L. (2005) Synthesis of selective SRPK-1 inhibitors: novel tricyclic quinoxaline derivatives. Bioorg. Med. Chem. Lett. 15, 3241-3246. 
(22) Morooka, S., Hoshina, M., Kii, I., Okabe, T., Kojima, H., Inoue, N., Okuno, Y., Denawa, M., Yoshida, S., Fukuhara, J., Ninomiya, K., Ikura, T., Furuya, T., Nagano, T., Noda, K., Ishida, S., Hosoya, T., Ito, N., Yoshimura, N., and Hagiwara, M. (2015) Identification of a Dual Inhibitor of SRPK1 and CK2 That Attenuates Pathological Angiogenesis of Macular Degeneration in Mice. Mol. Pharmacol. 88, 316325.

(23) Muller, S., Chaikuad, A., Gray, N. S., and Knapp, S. (2015) The ins and outs of selective kinase inhibitor development. Nat. Chem. Biol. $11,818-821$.

(24) Ghosh, G., and Adams, J. A. (2011) Phosphorylation mechanism and structure of serine-arginine protein kinases. FEBS J. 278, 587-597.

(25) Federov, O., Niesen, F. H., and Knapp, S. in Kinase Inhibitors: Methods and Protocols, Methods Mol. Biol., Vol. 795 (Kuster, B., Ed.), pp 109-118, Springer, 2011.

(26) Koeberle, S. C., Fischer, S., Schollmeyer, D., Schattel, V., Grütter, C., Rauh, D., and Laufer, S. A. (2012) Design, Synthesis, and Biological Evaluation of Novel Disubstituted Dibenzosuberones as Highly Potent and Selective Inhibitors of p38 Mitogen Activated Protein Kinase. J. Med. Chem. 55, 5868-5877.

(27) Siqueira, R. P., de Almeida Alves Barbosa, É., Polêto, M. D., Righetto, G. L., Seraphim, T. V., Salgado, R. L., Ferreira, J. G., de Andrade Barros, M. V., de Olivera, L. L., Laranjeira, A. B. A., Almeida, M. R., Júnior, A. S., Fietto, J. L. R., Kobarg, J., de Oliveira, E. B., Teixeira, R. R., Borges, J. C., Yunes, J. A., and Bressan, G. C. (2015) Potential Antileukemia Effect and Structural Analyses of SRPK Inhibition by $\mathrm{N}$-(2-(Piperidin-1-yl)-5-(Trifluoromethyl)Phenyl)Isonicotinamide (SRPIN340). PLoS One 10, e0134882.

(28) Saishin, Y., Saishin, Y., Takahashi, K., Lima e Silva, R., Hylton, D., Rudge, J. S., Wiegand, S. J., and Campochiaro, P. A. (2003) VEGFTRAP(R1R2) suppresses choroidal neovascularization and VEGFinduced breakdown of the blood-retinal barrier. J. Cell. Physiol. 195 (2), $241-8$.

(29) Hua, J., Spee, C., Kase, S., Rennel, E. S., Magnussen, A. L., Qiu, Y., Varey, A., Dhayade, S., Churchill, A. J., Harper, S. J., Bates, D. O., and Hinton, D. R. (2010) Recombinant human VEGF165b inhibits experimental choroidal neovascularization. Invest. Ophthalmol. Visual Sci. 51 (8), 4282-8.

\section{NOTE ADDED AFTER ASAP PUBLICATION}

This paper was published on February 6, 2017. The names of two of the authors (Roya Babaei-Jadidi and Jules C. Hancox) were incorrectly spelled. This has been corrected and the paper was re-posted on February 27, 2017. 